\title{
Effects of different vasopressors on the contraction of the superior mesenteric artery and uterine artery in rats during late pregnancy
}

\section{Tingting Wang}

Shanghai Changning Maternity and Infant Health Hospital

\section{Limei Liao}

Department of Anesthesiology and Perioperative Medicine, Guangzhou Women and Children's Medical Center, Guangzhou Medical University

\section{Xiaohui Tang}

Shanghai Changning Maternity and Infant Health Hospital

\section{Bin Li}

Shanghai Changning Maternity and Infant Health Hospital

Shaoqiang Huang ( $\nabla$ timrobbins71@163.com )

Obstetrics and Gynecology Hospital of Fudan University

\section{Research Article}

Keywords: Vasopressor, Mesenteric artery, Uterine artery, Contraction

Posted Date: March 17th, 2021

DOI: https://doi.org/10.21203/rs.3.rs-283519/v1

License: (c) (1) This work is licensed under a Creative Commons Attribution 4.0 International License. Read Full License 
Effects of different vasopressors on the contraction of the superior mesenteric artery and uterine artery in rats during late pregnancy

Tingting Wang ${ }^{1 *}$, Limei Liao ${ }^{2 *}$, Xiaohui Tang ${ }^{1}$, Bin Li $^{1}$, Shaoqiang Huang ${ }^{3}$

${ }^{1}$ Department of Anaesthesia, Changning Maternity and Infant Health Hospital, Shanghai, 200050, China.

${ }^{2}$ Department of Anesthesiology and Perioperative Medicine, Guangzhou Women and Children's Medical Center, Guangzhou Medical University, 9\# Jinsui Road, Guangzhou, 510623, China.

${ }^{3}$ Department of Anaesthesia, Obstetrics \& Gynecology Hospital, Fudan University, Shanghai, 200090, China

*These authors contributed equally to this work and should be considered co-first authors

Corresponding Author: Shaoqiang Huang, Department of Anaesthesia, Obstetrics \& Gynecology Hospital, Fudan University, 128\# Shenyang road, Shanghai 200090, China Email: timrobbins71@163.com, Phone: +86-21-63455050-6868; Fax: $+86-21-63455090$ 


\section{Abstract}

Background: Hypotension after neuraxial anaesthesia is one of the most common complications during caesarean section. Vasopressors are generally agreed to be the most effective way to improve hypotension, but it is unclear which of these drugs is best for caesarean section. We assessed the effects of vasopressors on the contractile response of uterine arteries and superior mesenteric arteries in pregnant rats, with the goal of identifying a drug that raises the blood pressure of the systemic circulation while minimally affecting the uterine and placental circulation.

Methods: Isolated ring segments from the uterine and superior mesenteric arteries of pregnant rats were mounted in organ baths, and their contractile responses to several vasopressor agents were studied. Concentration-response curves for norepinephrine, phenylephrine, metaraminol and vasopressin were constructed.

Results: The experimental results showed that the contractile response of the mesenteric artery to norepinephrine, as measured by the pEC50 of the drug, was stronger than that of the uterine artery $(5.617 \pm 0.11$ vs. $4.493 \pm 1.35, p=0.009)$, and the contractile response of the uterine artery to metaraminol was stronger than that of the mesenteric artery (pEC50: $5.084 \pm 0.17$ vs $4.92 \pm 0.10, p=0.007)$. There was no statistically significant difference in the pEC50 of phenylephrine or vasopressin between the two blood vessels.

Conclusion : In vitro experiments show that compared with phenylephrine, metaraminol, vasopressin, norepinephrine can contract peripheral blood vessel more strongly, while having the least effect on the contraction of uterine artery. These findings provide some support for the use of norepinephrine in mothers between the 
time of neuraxial anaesthesia and the delivery of the foetus.

Keywords: Vasopressor; Mesenteric artery; Uterine artery; Contraction 


\section{Background}

Hypotension after neuraxial anaesthesia is one of the most common complications during caesarean section. Without preventive treatment, the incidence of hypotension during caesarean section is as high as $80 \%$ [1]. Severe hypotension can cause nausea, vomiting, dizziness, confusion, and even cardiac arrest.

Methods to prevent and treat hypotension during caesarean section include volume supplementation before anaesthesia, reduction of anaesthetic doses and injection speeds, post-anaesthesia position adjustment and vasopressor usage. It is generally believed that the most effective way to improve hypotension is to use vasopressor agents [2]. Vasopressors commonly administered to pregnant women in clinical practice include phenylephrine, norepinephrine, metaraminol, and vasopressin. Each of these vasopressors has its own advantages and disadvantages, but the existing research is limited to the effect of vasopressors on maternal circulation changes and neonatal acidaemia; it is not clear whether vasopressors affect perfusion via the uterine artery (the largest visceral blood vessel that affects the foetus).The best vasopressor should effectively contract the peripheral blood vessels during the period after neuraxial anaesthesia and before the delivery of the foetus.

Phenylephrine, norepinephrine and metaraminol stimulate adrenaline receptors, and vasopressin exerts an increased blood pressure effect by stimulating $\mathrm{V}$ receptors on the cell membrane. However, the distribution and density of adrenaline and V receptor subtypes vary among different types of vascular beds, causing different blood vessels to have different vasoconstrictive responses to the same agents [3]. In addition, 
changes in hormone levels during pregnancy may affect the vasoconstrictive response to vasopressors. For example, Colucci et al. extracted blood vessels from male rats treated with $17 \beta$-oestradiol and found that the contractile response of the mesenteric arteries to norepinephrine was 4 times lower than that of non-oestradiol-treated rats [4]. Magness et al. measured the uterine blood flow and uterine vascular resistance of sheep after a bolus of norepinephrine and phenylephrine; they found that the effect of $\alpha$-adrenergic agonists on the uterine vascular system was weakened during pregnancy [5]. These studies suggest that the effects of various vasopressors on the uterine arteries and the smooth muscles of the systemic circulation differ between pregnant and non-pregnant states.

Considering that the relative efficacy of blood vessels is particularly related to the selection and dose determination of appropriate vasopressors, different blood vessels may respond differently to vasopressors. However, a current limitation on research in this area is that the extraction of human blood vessels is subject to many ethical restrictions. In view of the $90 \%$ genetic similarity between rats and humans, we chose to measure the isolated blood vessels of rats as a simulation of human vessels. In this study, we compare the effects of vasopressors on the contractile response of uterine arteries and superior mesenteric arteries in pregnant rats and then discuss the clinical implications of the vasopressor responses of isolated uterine arteries and systemic arteries. Through this study, we aim to compare treatment options and provide a basis for choosing the best vasopressor during the period between neuraxial anaesthesia and delivery of the foetus. 


\section{Methods}

\section{Laboratory animals}

The experimental animal programs involved in this experiment were all carried out in accordance with the basic principles of Fudan University's animal experiments, and have been approved by the Experimental Animal Committee of Fudan University.

After the protocol was approved by the Animal Ethics Committee of Shanghai Medical College of Fudan University (201907007Z), specific-pathogen-free (SPF) Sprague-Dawley rats (SD) (weighing 300-360 g at 16 days of pregnancy were selected and purchased from Shanghai Jie Sijie Laboratory Animal Co., Ltd. They were kept in an animal facility for 4 days and given a 12:12-hour light-dark cycle. The rats had free access to standard food and tap water. After 4 days, i.e., at 20 days of pregnancy, the rats were used for the experiment.

\section{Superior mesenteric artery and uterine artery acquisition}

Animals were euthanized using sodium pentobarbital $(100 \mathrm{mg} / \mathrm{kg}$, IP). After the death of the rat, the tissue was cut along the midline of the abdomen to fully open the abdominal cavity. The intestinal segment from the beginning of the duodenum to the end of the ileum was taken along with its mesangium, the uterine tissue and the communicating blood vessels between them. Two aliquots of physiological salt solution (PSS) were prepared, precooled to $4^{\circ} \mathrm{C}$, and fully oxygenated. The components of PSS were as follows (in mmol): $\mathrm{NaCl}, 118.3 ; \mathrm{KCl}, 4.7 ; \mathrm{MgSO}_{4}, 1.2$; $\mathrm{KH}_{2} \mathrm{PO}_{4}, 1.22 ; \mathrm{CaCl}_{2}, 2.5 ; \mathrm{NaHCO}_{3}, 25.0$; calcium ethylenediaminetetraacetate, 0.016; 
glucose, 11.1.

Under a dissecting microscope, remove the adipose tissue, connective tissue around the selected secondary/tertiary artery and the veins accompanying the artery. The clean arteries were separated and trimmed into two 3-mm-long vascular rings, which were threaded and fixed in a vascular tone measurement system [DMT 620M vascular tone measurement system (Denmark)] filled with $5 \mathrm{ml}$ of PSS solution at a constant temperature of $37^{\circ} \mathrm{C}$, and the solution was bubbled with $95 \%$ oxygen and $5 \%$ carbon dioxide. Care was taken throughout the process to avoid damaging the vascular endothelium during the whole process.

The fourth-order branches $(* 0.8 \mathrm{~mm}$ in external diameter) of the main uterine arteries were separated from the surrounding tissue and cut into 3-mm ring segments. The small branches of the uterine arteries were chosen because they are similar in nature to arterioles and play a substantial role in vascular resistance. The remaining steps were the same as those for the superior mesenteric artery.

\section{Measurement of vascular ring tension}

The transmural pressure of the superior mesenteric artery and uterine artery was adjusted to balance under basal tension of $3 \mathrm{mN}$ for 1 hour. After calibration to zero, potassium chloride $(\mathrm{KCl})$ was administered twice to stimulate vasoconstriction. Each stimulation used $60 \mathrm{mM} \mathrm{KCl}$ and lasted 10 minutes. After the contraction tension was stabilized, the average contraction strength was recorded and calculated (i.e., $\Delta \mathrm{KCl}=$ $\Sigma$ (peak contraction response - base tension value before contraction)/2). Then, the 
cells were rinsed with PSS 3 times for 10 minutes each to restore the contraction curve to the baseline level.

The integrity of vascular endothelial function was tested by adding gradually increasing concentrations of phenylephrine $\left(\mathrm{PE}, 10^{-9}\right.$ to $\left.10^{-4} \mathrm{M}\right)$ into the bath of the vascular tension measurement system to cause maximum contraction, then adding gradually increasing concentrations of acetylcholine (ACh, $10^{-9}$ to $10^{-4} \mathrm{M}$ ) to reduce the vascular tension to the lowest value and stabilize it at that value. If the decrease in vascular tension was less than $10 \%$ of the maximum contraction caused by PE, endothelial function was considered to be lost; if the decrease in vascular tension was greater than $80 \%$ of the maximum contraction caused by PE, endothelial function was considered intact; and if the vascular tension drops between $10 \%$ and $80 \%$, the endothelium was considered damaged [6]. In this experiment, only vascular rings with intact endothelium were used.

After testing the integrity of arterial endothelial function, we waited for the vascular tension curve to stabilize at the baseline level and added gradually increasing concentrations of vasopressors (phenylephrine, norepinephrine, vasopressin, metaraminol) to the water bath $\left(10^{-9}-10^{-5} / 10^{-4} \mathrm{M}\right)$. Each drug concentration was allowed to for 2 minutes to ensure that the vascular tension reaches a stable level under the drug concentration. The contraction strength was observed and recorded at each concentration (i.e., $\Delta$ contraction strength $=$ peak contraction response - baseline tension value). After stimulation was completed with the highest drug concentration, the vascular ring was washed twice with PSS solution for 5 minutes each, and then the 
activity of the vascular ring was measured during stimulation with $60 \mathrm{mM} \mathrm{KCl}$ (Figure 1).

Vascular rings were excluded if they did not respond to high potassium stimulation before or after the experiment or if they had incomplete endothelial function.

\section{Statistical analysis}

In order to reflect whether the sample average can replace the overall average, all data are expressed as the mean \pm standard error (SE) of $n$ experiments. The maximum tension $(\mathrm{mN})$ of the vascular ring was recorded at each drug concentration, and the ratio of the maximum tension at the drug concentration to the average value of the maximum tension obtained from two potassium chloride stimulations was calculated and defined as the KPSS\% of the vascular ring. With KPSS\% as the ordinate and the logarithm of the vasopressor concentration as the abscissa, GraphPad Prism 8 was used to fit the concentration-contraction effect curve. Using SPSS Statistics 22 software, Student's t-test (if applicable) or a non-parametric test was used to compare the power of EC50 (the molar concentration required to cause $50 \%$ of the maximum response) and the maximum response between the drugs. In all cases, P (two-tailed) $<0.05$ was considered statistically significant. 


\section{Results}

A total of 10 rats at 20 days of pregnancy were included in this study, and one uterine artery ring and one superior mesenteric artery ring were extracted from each rat. In the uterine artery, the maximum response of KPSS is $100 \%$, and the Emax range of each of the four contractile drugs is $65 \%$ to $109 \%$ of KPSS. The maximum contraction of vasopressin was significantly higher than that of norepinephrine $(108.51 \pm 21.07$ vs $82.73 \pm 36.21 \%, \mathrm{p}=0.031)$, and the maximum contraction of phenylephrine and metaraminol was not significantly different from that of norepinephrine (Table 1). In the pEC50 comparison, vasopressin was significantly higher than norepinephrine $(7.87 \pm 0.56$ vs $4.493 \pm 1.35, \mathrm{p}<0.001)$; the maximum contraction of phenylephrine and metaraminol was not significantly different from that of norepinephrine (Table 1).

In the mesenteric artery, the Emax of phenylephrine, norepinephrine, metaraminol and vasopressin were $76 \pm 26 \%, 83 \pm 17 \%, 62 \pm 26 \%, 70 \pm 33 \%$, respectively, of which norepinephrine was significantly higher than metaraminol $(\mathrm{P}=0.029)$ (Figure 2 and Table 1). In comparison of pEC50, norepinephrine was significantly higher than phenylephrine and metaraminol $(5.617 \pm 0.11$ vs. $5.252 \pm 0.06, \mathrm{p}<0.001 ; 5.617 \pm 0.11$ vs. $4.92 \pm 0.10, \mathrm{p}<0.001)$, and vasopressin was significantly higher than norepinephrine $(7.958 \pm 0.38$ vs. $5.617 \pm 0.11, \mathrm{p}<0.001)$.

Comparing the pEC50 of the four blood pressure drugs in different blood vessels, norepinephrine in the mesenteric artery contracted more strongly than that in the uterine artery $(5.617 \pm 0.11$ vs $4.493 \pm 1.35, \mathrm{p}=0.009)$, and the contraction of 
metaraminol in the uterine artery was stronger than that in the mesenteric artery (5.084 \pm 0.17 vs $4.92 \pm 0.10, \mathrm{p}=0.007)$. There was no significant difference in the pEC50 between phenylephrine and vasopressin in the two blood vessels $(5.223 \pm 0.083$ vs $5.252 \pm 0.06, \mathrm{p}=0.325 ; 7.87 \pm 0.56$ vs $7.958 \pm 0.38, \mathrm{p}=0.649)$ (Table 2 ). 


\section{Discussion}

To the best of our knowledge, no published study has simultaneously investigated the efficacies of these vasopressors in blood vessels from different parts of the body. This is the first study to directly compare the efficacy of vasopressors used in isolated uterine arteries and mesenteric arteries in pregnant mice. The results of the study showed that the contraction of norepinephrine in the mesenteric artery was stronger than that of the uterine artery $(5.617 \pm 0.11$ vs. $4.493 \pm 1.35, \mathrm{p}=0.009)$, and the contraction of metaraminol in the uterine artery was stronger than that of the mesenteric artery $(5.084 \pm 0.17$ vs $4.92 \pm 0.10), \mathrm{p}=0.007)$. There was no statistically significant difference between the pEC50 of phenylephrine and vasopressin in the two blood vessels. In the period between neuraxial anaesthesia and the delivery of the foetus, the optimal vasopressors should constrict the peripheral blood vessels while having the least impact on the contraction of the uterine artery. Therefore, the results of the in vitro experiment suggest that norepinephrine may be the most suitable vasopressor.

The uterine artery is the main source of uterine blood during pregnancy, carrying $11 \%$ of the total cardiac output in late pregnancy [7]. This vessel is the main site where materials are exchanged to and from the foetus. However, uterine blood flow cannot be adjusted in isolation [8]. If the blood flow of the uterine artery is excessively reduced, the oxygen supply to the foetus in the uterus is reduced, and the central nervous system may be damaged, which poses a serious risk of foetal death. Therefore, for the parturient, the best vasopressor should effectively contract the 
peripheral blood vessels during the period after neuraxial anaesthesia and before the delivery of the foetus. Such an agent should maximize the stability of the peripheral circulation while minimally affecting the blood pressure in the uterine artery and maintaining the uterine blood supply and oxygenation, thereby ensuring the safety of mothers and newborns.

Currently, the most appropriate vasopressor remains controversial during caesarean section. Phenylephrine is a pure $\alpha 1$ receptor agonist. Traditionally, it has the advantages of fast onset, no passage through the placenta, and stable foetal acid-base balance. However, studies in the past five years have found that phenylephrine has the risk of reducing maternal cardiac output and heart rate, so the safety of phenylephrine has been questioned. In 2015, Ngan Kee et al. proposed that norepinephrine has a $\beta$-receptor excitatory effect in addition to its potent $\alpha$-receptor stimulus, which can improve maternal cardiac output and heart rate [9]. Therefore, it is considered to replace phenylephrine as the first drug for caesarean section to prevent and treat hypotension. In addition, some studies believe that metaraminol is better than ephedrine in terms of foetal academia [10], but it lacks the comparison of metaraminol and other vasoactive drugs on circulation and visceral blood vessels. Vasopressin binds to the vasopressin (V) receptor on the cell membrane to increase water permeability and increase circulating blood volume. Some studies believe that vasopressin in the same concentration range effectively shrinks the radial artery while having no contraction effect on the pulmonary artery [11]. However, there is no relevant study comparing the contractile efficacy of vasopressin in the same 
concentration range in the systemic circulation and uterine artery.

Pregnancy is associated with a marked increase in uterine blood flow [12], which optimizes the delivery of oxygen and substrates through the placenta to the developing foetus [13]. The adaptation of the uterine circulation to pregnancy is complex and partly mediated by enhanced vasodilation and vascular remodelling [14]. The adaptability of the smooth muscle contraction mechanism is unclear. Pressure-dependent myogenic contraction may be an important physiological mechanism for regulating basal vascular tension and an important factor for regulating blood flow [15]. Therefore, the contractile efficacy of blood vessels has a direct effect on blood flow regulation.

Adrenergic receptors are recognized as the cell membrane binding sites through which natural catecholamines, namely, adrenaline (epinephrine) and noradrenaline (norepinephrine), exert their physiological effects on target tissues of mammals [16]. These neurotransmitters have several different actions on organisms, particularly on the cardiovascular system, smooth muscle and adipose tissue, by activating specific proteins on the surface of the plasma membrane (adrenergic receptors) [17]. Studies have shown that there are $\alpha 1 \mathrm{~A}, \alpha 1 \mathrm{~B}$, and $\alpha 1 \mathrm{D}$ receptors on the mesenteric arteries of rats and humans, and the numbers of receptors will change with age [18].

Pregnancy can lead to enhanced endothelium-dependent relaxation, inherent changes in vascular smooth muscle or changes in the vascular adrenergic response, thereby changing the vascular response. Both $\alpha$ - and $\beta$-adrenergic regulation are important mechanisms in the control of uterine blood flow and foetal oxygenation 
[19]. Studies suggest that contraction to $\alpha$-adrenoceptor agonists in the late pregnant ovine uterine artery is mediated primarily by $\alpha 1$-adrenoceptors, and there appears to be no substantial $\alpha 1$-adrenoceptor reserve in this tissue [20]. Some scholars believe that the $\alpha 1$-adrenergic response is upregulated, whereas the $\beta$-adrenergic response is impaired, in the uterine microcirculation of pregnant rats [19]. Although the mechanism of action of adrenergic receptors during pregnancy is not clear, it is generally believed that pregnancy is associated with significant changes in the active contractile properties of uterine resistance artery function, specifically heightened $\alpha$-adrenergic sensitivity, intrinsic (pressure-dependent) tone, and myogenic reactivity [21]. Therefore, the vascular response to the drug during pregnancy is worthy of study and attention.

Although these four drugs are all vasopressors, they have different mechanisms of action. For example, norepinephrine and metaraminol act on receptors in a similar manner, acting mainly on $\alpha$ receptors and weakly on $\beta 1$ receptors. Phenylephrine mainly stimulates $\alpha 1$ receptors, with little or no $\beta$-type effect [22]. Vasopressin acts on $\mathrm{V} 2$ receptors on the peritubular membrane of epithelial cells [23, 24]. A-adrenergic receptor-mediated contraction of vascular smooth muscle plays an important role in controlling peripheral circulation. The $\alpha 1$-adrenergic receptor is a $\mathrm{Gq} / 11$ protein-coupled receptor. $\alpha 1$-Adrenergic receptor $(\alpha 1 \mathrm{AR})$ stimulation mediates sympathetic nervous system responses, such as vascular smooth muscle contraction and cardiac hypertrophy. $\alpha 1 \mathrm{AR}$-mediated vasoconstriction contributes to baseline (tonic) vascular tension and regulates systemic vascular resistance/venous volume. 
After activating the $\alpha 1$-adrenergic receptor, the heterotrimeric $G$ protein $G q / 11$ dissociates to release the $\alpha$ and $\beta \gamma$ subunits, which in turn activates phosphoinositide C (PLC) to induce the phosphoinositide signal cascade. PLC activation leads to an increase in intracellular IP3, which binds to IP3 receptors on the endoplasmic reticulum, causing calcium to be released from intracellular storage, followed by a massive influx of calcium, and finally triggers smooth muscle contraction [25].

This study provides a reference for clinical application and reminds anaesthesiologists and clinicians to consider the effects of drugs on uterine artery contraction when using vasopressors. During caesarean section, especially during the period before delivery of the foetus after neuraxial anaesthesia, there is an extremely high probability that the patient will need vasopressors. Incontinence or excessive use may cause overdone contraction of the uterine artery, resulting in decreased placental perfusion and even adverse effects on the foetus. At present, the choice of vasopressor is controversial. This study provides a perspective suggesting that norepinephrine may be the most suitable vasopressor for women undergoing caesarean section.

The current study has the following limitations. 1. The content and ratio of adrenergic receptor subtypes in different species may vary. The properties and the distribution of the subtypes differ among species, all suggesting that the pharmacodynamic properties of an adrenergic compound may significantly change according to the tissues and the animal species. However, it is very difficult to collect maternal uterine artery and mesenteric artery clinically, and the in vitro experiment is very mature and can intuitively compare the performance, so it is the more suitable 
comparison method. 2. The study does not combine in vitro experiments with clinical trials. In vitro experiments study the direct effects of drugs on vascular smooth muscle, but in addition to the direct effects of drugs, the body is regulated by nerves and body fluids, which is a complex whole. 3. The specific mechanism of blood vessel contraction by vasopressors has not been further explored.

In summary, this study showed that norepinephrine elicits stronger contractions in the mesenteric artery than in the uterine artery in vitro. In contrast, the contractile response to metaraminol was stronger in the uterine artery than in the mesenteric artery. There was no significant difference in the pEC50 of phenylephrine or vasopressin between the two blood vessels. The results of in vitro experiments suggest that norepinephrine may be the most suitable vasopressor for the period between neuraxial anaesthesia and the delivery of the foetus. 
Figue 1. Experimental design. Adjust the transmural pressure of superior mesenteric artery and uterine artery to balance under the basal tension of $3 \mathrm{mN}$ for 1 hour. After zero adjustment, $60 \mathrm{mM}$ potassium chloride (KCL) was implemented twice to stimulate vasoconstriction for 10 minutes. Test the integrity of vascular endothelial function (phenylephrine, PE, $10^{-9}$ to $10^{-4} \mathrm{M}$; acetylcholine , $\mathrm{ACH}, 10^{-9}$ to $10^{-4} \mathrm{M}$ ). Then added gradually increasing concentrations of vasopressor (phenylephrine, norepinephrine, vasopressin, metaraminol) in the water bath $\left(10^{-9}-10^{-5} / 10^{-4} \mathrm{M}\right)$. Each drug concentration acts for 2 minutes.

Figure 2. Contractile responses to uterotonic agents in rat uterine arteries and mesenteric arteries. Cumulative concentration--response curves to phenylephrine $\left(10^{-9} \sim 10^{-4} \mathrm{M}\right)$, norepinephrine $\left(10^{-9} \sim 10^{-4} \mathrm{M}\right)$, metaraminol $\left(10^{-9} \sim 10^{-4} \mathrm{M}\right)$, or vasopressin $\left(10^{-9} \sim 10^{-5} \mathrm{M}\right)$ were constructed. Contractile responses are shown as a percentage of KPSS (potassium depolarizing solution) maximum contraction. 


\section{Ethics approval and consent to participate:}

All animal procedures were approved (201907007Z) by the animal ethics committee of Shanghai Medical College of Fudan University and were performed in strict accordance with recommendations of the Guide to the Care and Use of Laboratory Animals of the National Institutes of Health. Our manuscript reporting adheres to the ARRIVE guidelines in accordance with BioMed Central editorial policies.

\section{Funding:}

Special project of medical and health research in Changning District of Shanghai in 2020, CNKW2020Y28;

Xinchen cup young anesthesiologist training fund of anesthesia branch of Shanghai Medical Association;

Changning District Health Commission Medical Specialty 2019, 20192003

\section{Availability of data and materials}

The data sets used and/or analyzed during the current study are available from the corresponding author on reasonable request.

\section{No any conflict of interest}

\section{Author's Contribution}

T.T.W.: study design, data collection, data analysis and interpretation of the results, and writing of the paper; L.M.L.: study design, data collection, data analysis and 
interpretation of the results, and writing of the paper; B.L.: data collection, data analysis; X.H.T.: data collection, data analysis; S.Q.H.: study design, data analysis, interpretation of the results, and writing of the paper.

\section{Consent for publication}

Not applicable.

\section{Author details}

1 Department of Anaesthesia, Changning Maternity and Infant Health Hospital, Shanghai, 200050, China. ${ }^{2}$ Department of Anesthesiology and Perioperative Medicine, Guangzhou Women and Children's Medical Center, Guangzhou Medical University, 9\# Jinsui Road, Guangzhou, 510623, China.

${ }^{3}$ Department of Anaesthesia, Obstetrics \& Gynecology Hospital, Fudan University, Shanghai, 200090, China 


\section{Reference}

1. S Saravanan, M Kocarev, R C Wilson, E Watkins, M O Columb, G Lyons:

Equivalent dose of ephedrine and phenylephrine in the prevention of post-spinal hypotension in caesarean section. Br J Anaesth 2006; 96(1): 95-99.

2. Ngan Kee WD, Khaw KS: Vasopressom in obstetrics: what should we be using? Curr Opin Anaesthesiol 2006;19(3): 238-243.

3. Rudner XL, Berkowitz DE, Booth JV, Funk BL, Cozart KL, D’Amico EB, El-Moalem H, Page SO, Richardson CD, Winters B, Marucci L, Schwinn DA: Subtype specific regulation of human vascular alpha (1)-adrenergic receptors by vessel bed and age. Circulation 1999; 100:2336-43

4. Colucci WS, Gimbrone MA Jr, McLaughlin MK, Halpern W, Alexander RW: Increased vascular catecholamine sensitivity and alpha-adrenergic receptor affinity in female and estrogen-treated male rats. Circ Res 1982; 50(6):805-11

5. Magness RR, Rosenfeld CR: Systemic and uterine responses to alpha-adrenergic stimulation in pregnant and nonpregnant ewes. Am J Obstet Gynecol 1986; 155(4):897-904.

6. Gustavo Ballejo, Tiago A Barbosa, Eduardo B Coelho, Cristina Antoniali, Maria Cristina O Salgado: Pregnancy-associated increase in rat systemic arteries endothelial nitric oxide production diminishes vasoconstrictor but does not enhance vasodilator responses. Life Sci.2002; 70(26):3131-42.

7. R A Ahokas, G D Anderson, J Lipshitz. Cardiac output and uteroplacental blood 
flow in diet-restricted and diet-repleted pregnant rats. Am J Obstet Gynecol. 1983; 146: 6-13.

8. Birnbach DJ,Browne IM.Anesthesia for obstetrics. In: Miller RD, Eriksson LI, Fleisher LA, Wiener-Kronish JP, Young WL. Miller's Anesthesia.7th ed. Philadelphia, PA: Churchill Livingstone: 2009; 2203-2240.

9. Warwick D Ngan Kee, Shara W Y Lee, Floria F Ng, Perpetua E Tan, Kim S Khaw: Randomized double-blinded comparison of norepinephrine and phenylephrine for maintenance of blood pressure during spinal anesthesia for cesarean delivery. Anesthesiology. 2015 ; 122(4):736-45.

10. Ngan Kee WD, Lau TK, Khaw KS, Lee BB: Comparison of metaraminol and ephedrine infusions for maintaining arterial pressure during spinal anesthesia for elective cesarean section. Anesthesiology 2001; 95:307-313.

11. Dale A Currigan, Richard J A Hughes, Christine E Wright, James A Angus, Paul F Soeding: Vasoconstrictor responses to vasopressor agents in human pulmonary and radial arteries: an in vitro study. Anesthesiology. 2014; 121(5):930-6.

12. Juanjuan Chen, Raouf A Khalil : Matrix Metalloproteinases in Normal Pregnancy and Preeclampsia. Prog Mol Biol Transl Sci 2017;148 :87-165.

13. Ishmael M Inocencio, Graeme R Polglase, Ilias Nitsos, Suzanne L Miller, Beth J Allison : Maternal sildenafil impairs the cardiovascular adaptations to chronic hypoxaemia in fetal sheep. J Physiol. 2020 ; 598(19):4405-4419.

14. Zuzana Adamova, Sifa Ozkan, Raouf A Khalil: Vascular and cellular calcium in normal and hypertensive pregnancy .Curr Clin Pharmacol. 2009 ; 4(3):172-90. 
15. Michael J Davis: Perspective: physiological role(s) of the vascular myogenic response. Microcirculation. 2012; 19(2):99-114.

16. $\mathrm{P}$ Badino, $\mathrm{R}$ Odore, $\mathrm{G}$ Re: Are so many adrenergic receptor subtypes really present in domestic animal tissues? A pharmacological perspective. Vet J. 2005; 170 (2):163-74.

17. P B Timmermans, $\mathrm{P}$ A van Zwieten: alpha 2 adrenoceptors: classification, localization, mechanisms, and targets for drugs. J Med Chem. 1982 ; 25(12):1389-401.

18. X L Rudner, D E Berkowitz, J V Booth, B L Funk, K L Cozart, E B D'Amico, H El-Moalem, S O Page, C D Richardson, B Winters, L Marucci, D A Schwinn: Subtype specific regulation of human vascular alpha(1)-adrenergic receptors by vessel bed and age. Circulation.1999; 100(23):2336-43.

19. Steven Y Wang, Sanjay Datta, Scott Segal: Pregnancy alters adrenergic mechanisms in uterine arterioles of rats. Anesth Analg. 2002; 94(5):1304-9.

20. M Isla, D C Dyer: Characterization of alpha-adrenoceptors in the late pregnant ovine uterine artery . Eur J Pharmacol. 1990; 178 (3):321-31.

21. G Osol, M Cipolla: Interaction of myogenic and adrenergic mechanisms in isolated, pressurized uterine radial arteries from late-pregnant and nonpregnant rats. Am J Obstet Gynecol. 1993; 168 (2):697-705.

22. Cooper DW: Caesarean delivery vasopressor management. Curr Opin Anaesthesiol. $2012 ; 25(3): 300-8$.

23. Treschan TA, Peters J: The vasopressin system: Physiology and clinical strategies. 
Anesthesiology 2006; 105:599-612

24. Dünser MW, Lindner KH, Wenzel V: A century of arginine vasopressin research leading to new therapeutic strategies. Anesthesiology 2006; 105:444-5

25. Chen ZJ, Minneman KP. Recent progress in alpha1-adrenergic receptor research. Acta Pharmacol Sin. 2005 ; 26(11):1281-7. 
Figures

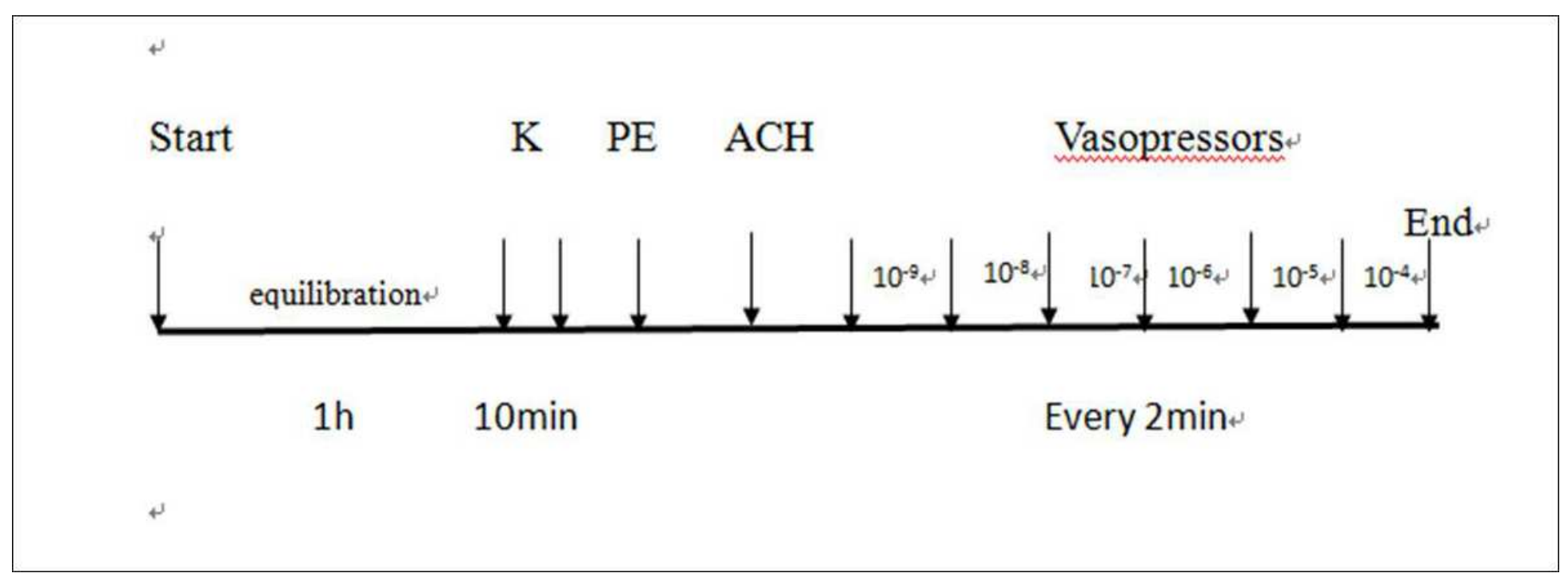

\section{Figure 1}

Experimental design. Adjust the transmural pressure of superior mesenteric artery and uterine artery to balance under the basal tension of $3 \mathrm{mN}$ for 1 hour. After zero adjustment, $60 \mathrm{mM}$ potassium chloride $(\mathrm{KCL})$ was implemented twice to stimulate vasoconstriction for 10 minutes. Test the integrity of vascular endothelial function (phenylephrine , $\mathrm{PE}, 10-9$ to $10-4 \mathrm{M}$; acetylcholine , $\mathrm{ACH}, 10-9$ to 10-4 M ). Then added gradually increasing concentrations of vasopressor (phenylephrine, norepinephrine, vasopressin, metaraminol) in the water bath (10-9-10-5/10-4 M). Each drug concentration acts for 2 minutes. 

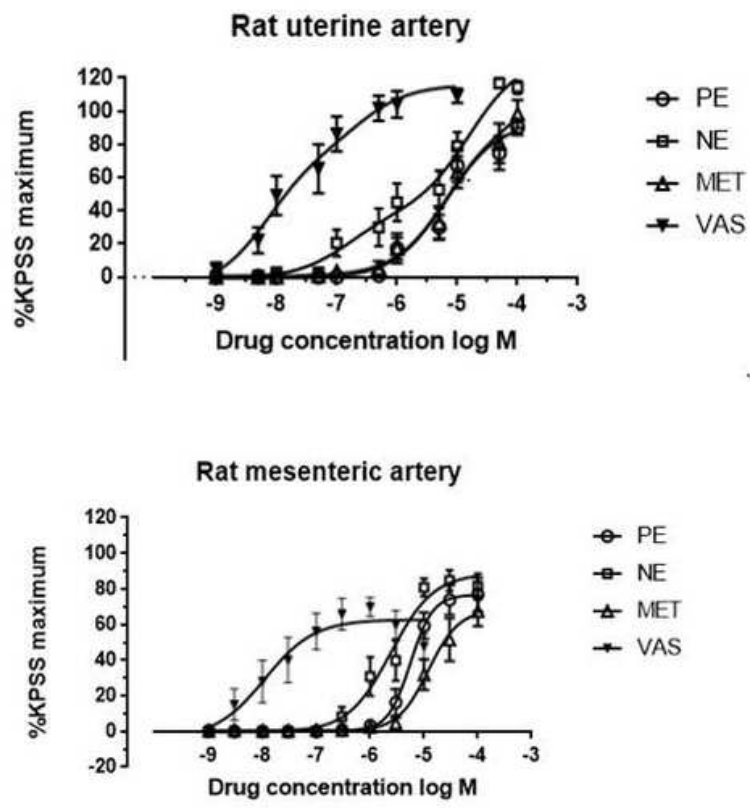

Figure 2

Contractile responses to uterotonic agents in rat uterine arteries and mesenteric arteries. Cumulative concentration-response curves to phenylephrine (10-9 10-4M), norepinephrine (10-9 10-4M), metaraminol (10-9 10-4M), or vasopressin (10-9 10-5M) were constructed. Contractile responses are shown as a percentage of KPSS (potassium depolarizing solution) maximum contraction. 